\title{
Snake-based interactive tooth segmentation for 3D mandibular meshes (erratum)
}

Rui Yang, Amir Abdi, Atabak Eghbal, Edward Wang, Khanh Linh Tran, et al.

Rui Yang, Amir H. Abdi, Atabak Eghbal, Edward Wang, Khanh Linh Tran, David Yang, Antony Hodgson, Eitan Prisman, Sidney Fels, "Snake-based interactive tooth segmentation for 3D mandibular meshes (erratum)," Proc. SPIE 11598, Medical Imaging 2021: Image-Guided Procedures, Robotic Interventions, and Modeling, 1159820 (13 May 2021); doi: $10.1117 / 12.2601031$

SPIE. Event: SPIE Medical Imaging, 2021, Online Only 


\title{
Snake-based interactive tooth segmentation for 3D mandibular meshes (Eratum)
}

\author{
Rui Yang, ${ }^{1}$ Amir H. Abdi, ${ }^{1}$ Atabak Eghbal, ${ }^{1}$ Edward Wang, ${ }^{1}$ Khanh Linh Tran, ${ }^{1}$ David \\ Yang, ${ }^{2}$ Antony Hodgson, ${ }^{1}$ Eitan Prisman, ${ }^{1}$ Sidney Fels ${ }^{1}$ \\ ${ }^{1}$ Univ. of British Columbia (Canada) \\ ${ }^{2} \mathrm{BC}$ Cancer (Canada) \\ Proceedings Volume 11598, Medical Imaging 2021: Image-Guided Procedures, Robotic Interventions, \\ and Modeling; 1159810 (2021) https://doi.org/10.1117/12.2581988
}

Event: SPIE Medical Imaging, 2021, Online Only

Online Publication Date: 15 February 2021

Enatum Published: 13 May 2021

A revised version of this manuscript was published on 13 May 2021. Details of the revision a re provided in the text that accompanies this Erratum. The original paper has been updated.

1. In the original version on page 9, Table 3, the standard deviation (SD) value of Symmetric RMSD for Group P-A was 0.34. It is corrected to 0.35. The SD value of Symmetric HD95 for Group M-M was 0.27. It is corrected to 0.40 .

2. In the original version, the description of the proposed method being "semi-automatic" was inaccurate. The term "semi-automatic" is removed in the new version. 